\title{
Anal intraepithelial neoplasia: diagnosis, screening, and treatment
}

\author{
Ragavan V. Siddharthan ${ }^{\mathrm{a}}$, Christian Lanciault ${ }^{\mathrm{b}}$, Vassiliki Liana Tsikitis ${ }^{\mathrm{a}}$ \\ Orgeon Health and Science University, Portland, OR, USA
}

Abstract

Anal intraepithelial neoplasia (AIN) is a premalignant lesion for anal cancer. It is more commonly
found in high-risk patients (e.g., human papilloma virus (HPV)/human immunodeficiency virus
infections, post-organ transplantation patients, and men who have sex with men) and development
is driven by HPV infection. The incidence of AIN is difficult to estimate, but is heavily skewed by
preexisting conditions, particularly in high-risk populations. The diagnosis is made from cytology
or biopsy during routine examinations, and can be performed at a primary care provider's office.
A pathologist can then review and classify cells, based on nucleus-to-cytoplasm ratios. The
classification of low or high grade can better predict progression from AIN to anal cancer. There
is little debate that AIN can develop into anal cancer, and the main rationale for treatment is
to delay the progression. Significant controversy remains regarding screening, surveillance, and
treatment for AIN. Management options are separated into surveillance (watchful waiting) and
interventional strategies. Emerging data suggest that close patient follow up with a combination
of ablative and topical treatments may offer the greatest benefit. HPV vaccination offers a unique
treatment prior to HPV infection and the subsequent development of AIN, but its use after the
development of AIN is limited. Ablative treatment includes excision, fulguration, and laser therapy.

Keywords Anal intraepithelial neoplasia, human papilloma virus (HPV), HPV-related squamous epithelial dysplasia

Ann Gastroenterol 2019; 32 (2): 1-7

\section{Introduction}

Human papilloma virus (HPV)-related squamous epithelial dysplasia is a precursor to invasive squamous cell carcinoma in multiple anatomic sites, including the anogenital tract. The Lower Anogenital Squamous Terminology Standardization (LAST) project for HPV-associated lesions recently provided guidelines for a unified nomenclature. A 2-tiered system, designated as low- or highgrade squamous intraepithelial lesion (LSIL or HSIL, respectively), is the recommended terminology for reporting. In the anal canal, LSIL correlates with anal intraepithelial neoplasia (AIN)-1 or anal condylomata, while HSIL correlates with AIN-2 and AIN-3. The 2-tiered system, based on consensus evaluation, more accurately reflects the biology of these HPV-related lesions [1].

Department of aSurgery, Division of Gastrointestinal and General Surgery (Ragavan V. Siddharthan, V. Liana Tsikitis); ${ }^{\text {PPathology }}$ (Christian Lanciault), Oregon Health and Science University, Portland, OR, USA

Conflict of Interest: None

Correspondence to: Vassiliki Liana Tsikitis, MD, MCR, FACS, FASCRS, 3181 SW Sam Jackson Park Road, Portland, Oregon 97239, USA, e-mail: tsikitis@ohsu.edu

Received 1 December 2018; accepted 24 January 2019; published online 18 February 2019

DOI: https://doi.org/10.20524/aog.2019.0364
A squamous intraepithelial anal lesion is the dysplastic growth of squamous epithelial cells in the transition zone of the anal canal. These lesions are pre-malignant, and can progress to anal squamous cell carcinoma if left untreated [2]. For ease of terminology in this paper, we will refer to LSIL lesions as AIN-1, and HSIL lesions as AIN-2/AIN-3, or high-grade AIN. AIN has a clear association with HPV, and is more prevalent in at-risk populations, such as human immunodeficiency virus (HIV)-positive patients, men who have sex with men (MSM), and renal transplant patients. In these populations, the rates of anal cancer are dramatically elevated, despite it being a relatively uncommon disease [3]. This review aims to explore the epidemiology, current strategies for diagnosis and treatment, and prognosis of AIN.

\section{Incidence}

The incidence of AIN within the general population is difficult to estimate. Rates of anal cancer are low, with approximately 1.8 cases per 100,000 men and women. This number is heavily skewed by preexisting conditions, particularly in high-risk populations, such as those infected with HPV or HIV, post-organ transplantation patients and MSM.

Several studies have tried to clarify the modern trends in anal cancer and AIN. Overall, prior to the 1990s, rates of 
anal cancer were higher in women than men, while in recent years, the rates have been comparable. Women have a higher incidence of anal cancer after age 50; the majority of patients, however, are men between the ages of 20-49 [4]. A large study from Denmark reviewed data from 1978-2008 to characterize the burden of disease at a national level. Comparing pre1980s data to post-2003 data, they found that the incidence of anal cancer increased for both men and women. They also discovered a $5 \%$ annual increase in the incidence of highgrade AIN between 1998 and 2008. The annual increase in AIN was larger than the annual increase in anal cancer, probably reflecting higher rates of screening; regardless, the scope of disease was larger than previously thought [5].

The rate of conversion of AIN to anal cancer remains controversial. Preliminary prospective studies suggest the rate is similar to that for cervical cancer, with about 9-13\% conversion for AIN-3 within a 5-year period for untreated patients [6,7], but there is variability among different risk populations, with higher rates in HIV-positive and immunocompromised patients. Despite these prospective studies, a large meta-analysis from 2012 found that the rate of conversion was much lower, with progression rates of 1 in 600 per year for HIV-positive MSM and 1 in 4000 per year for HIV-negative MSM patients [8]. This discrepancy may be attributed to the mix of AIN-2 and AIN-3 patients within the meta-analysis, while the prospective studies followed only AIN-3. Spontaneous regression of AIN occurs in a proportion of patients. A retrospective study showed that high-grade AIN regresses to AIN-1 at a rate of $17 \%$, and to no disease at a rate of $7 \%$. These patients were a mixture of HIV-positive and HIVnegative [9].

\section{Pathogenesis of disease and risk factors}

\section{HPV}

HPV is a sexually transmitted virus that currently infects an estimated 79 million Americans. Approximately 14 million people are infected every year; it is generally agreed that most sexually-active males and females will acquire an HPV infection at some point in their lifetime, though $90 \%$ of them will be silent and resolve spontaneously [10]. HPV is a nonenveloped double-stranded DNA virus that infects mucosal and cutaneous keratinocytes. The risk factors associated with persistent HPV infection are presented in Table 1 [11]. There are over 180 subtypes of the virus, 30 of which are thought to infect the anogenital tracts. These subtypes can usually be categorized into high- vs. low-risk in terms of their oncologic potential. HPV infection has been established as a leading cause of cancer and its causative effect in cervical cancer has been firmly established [12].

Retrospective analyses have shown HPV to have a clear association with AIN and anal cancer. HPV prevalence among patients with AIN is over 90\% [13]. A large meta-analysis that compared cervical cancer, vulvar carcinoma and anal cancer found that patients with anal cancer had the highest prevalence
Table 1 Risk factors for persistent HPV infection [11]

\begin{tabular}{ll}
\hline Behaviors & Multiple sexual partners \\
& Men who have sex with men \\
& Limited medical care \\
& Smoking \\
Medications & Oral contraception use \\
Immunosuppression & No HPV vaccination \\
Co-infections & HIV \\
& $\begin{array}{l}\text { Multiple HPV subtype infections } \\
\text { Other sexually transmitted infections }\end{array}$ \\
HPV infection characteristic & $\begin{array}{l}\text { High-risk HPV type 16, 18 } \\
\text { Viral load }\end{array}$ \\
\hline
\end{tabular}

HPV, human papilloma virus; HIV, human immunodeficiency virus

of HPV infection (84.3\%) [14]. In addition, the amount of HPV DNA found in biopsy specimens is higher for highergrade AIN than for lower-grade AIN, suggesting a role in the development of malignancy [15].

Though patients considered high-risk (MSM, HIV-positive, immunocompromised patients) often have HPV infection, it can occur in both heterosexual and non-HIV-infected patients. Multiple studies have found HPV in the anal canal of heterosexual men, with rates ranging between $8-30 \%$. For HIV-negative women, the prevalence of anal HPV approaches $27 \%$ [16]. These studies suggest that anal HPV is much more prevalent than previously thought.

The correlation between AIN and HPV is strong, but recent data has shown HPV may be causative for the development of AIN. Early microscopic studies demonstrated HPV particles within malignant cells and recent PCR studies have shown a large quantity of viral DNA within tumor cells $[17,18]$. The biologic mechanism for malignant transformation of cells is mediated by HPV's ability to encode tumor suppressor proteins E5, E6, and E7, which change the intracellular expression of p53 and retinoblastoma protein, leading to changes in cell growth and apoptosis [19]. High-grade AIN and anal cancer are characterized by increased E-gene expression and a loss of L-gene expression (viral capsid gene) [7].

\section{Low-risk HPV}

The most common low-risk subtypes of HPV are 6 and 11, associated with AIN-1 and carry a lower risk of malignancy. The majority of HPV infections fall into this low-risk category. Epidemiologic studies have shown that HPV 6 is associated with $3 \%$ of anal cancers, but this is attributed to concomitant infection with the commonest high-risk subtypes of HPV. Despite the low risk of cancer, the disease burden of AIN-1 can be large. Patients often need local treatment and surveillance.

\section{High-risk HPV}

The most researched high-risk HPV subtypes are 16 and 18. High-risk HPV is associated with AIN-2 and AIN-3, and estimates attribute $80-90 \%$ of anal cancers to these high-risk 
strains, most commonly subtype 16 [20]. Subtype 18 , on the other hand, is found more commonly in cervical cancer and is associated with adenocarcinoma, as opposed to HPV 16, more commonly associated with squamous carcinoma [14]. Anal cancer associated with high-risk HPV is more commonly found within the anal canal, and is less likely to be associated with the perianal skin [21].

\section{HIV}

HIV infection remains a major public health concern and is associated with an increased incidence of AIN and anal cancer, particularly in MSM [22]. The exact rate of AIN among HIVpositive patients is not known, but rates of anal cancer are 30100 times higher than in the general population; among HIVpositive patients, $80 \%$ of anal cancers occur in MSM [22]. The higher incidence of AIN and anal cancer among HIV-positive patients is probably multifactorial, but is likely to reflect a high prevalence of coinfection with HPV. It is estimated that 7290\% of HIV-positive patients are infected with HPV and that $90 \%$ of HIV-positive patients with AIN have been infected with a high-risk subtype of HPV [23]. The pathophysiology of HPV infection among HIV-positive patients is due to immunodeficient patients being unable to prevent the propagation of the HPV proteins (E2, ZE6, E7) associated with high-grade dysplasia [24].

Over the last 30 years, highly active antiretroviral therapy (HAART)-based treatment has revolutionized the treatment of HIV, with improved mortality and morbidity; despite these improvements, however, the incidence of anal cancer has increased in HIV-positive patients [20]. Retrospective data have not been conclusive about the relationship between decreasing CD4 counts and the increased risk of anal cancer; some studies support the relationship [25] while others do not [26]. The benefits of HAART improve the overall survival of HIV-positive patients, but its specific effect on AIN and anal cancer is inconclusive.

\section{Other risk factors}

Medical immunosuppression in the post-solid organ transplant is associated with both AIN and anal cancer. A literature review from 2007 found that the prevalence of AIN within renal transplant patients was $20 \%$, while rates of anal HPV infection in these patients approached 50\%. The increased relative risk of anal cancer was $10 \%$ for renal transplant patients [27]. In addition to the presence of immunosuppression, its duration and intensity contribute to the increasing risk of anal cancer and AIN [28].

Immunosuppression in other diseases, including inflammatory bowel disease (IBD) has been previously thought to contribute to AIN. Crohn's disease patients have been found to have both higher rates and an earlier onset of anal cancer [29]. However, a retrospective review of IBD patients found that the rates of AIN were similar, regardless of immunosuppressive status [30].

\section{Pathology}

The pathologic assessment of AIN has gone through several iterations. The most widely accepted classification is based on the LAST project, and separates the specimens into 2 tiers. The first tier is LSIL, which includes AIN-1, and the second is HSIL, which encompasses AIN-2 and AIN-3. The histologic characteristics of LSIL are superficial cell atypia with nuclear enlargement and nuclear membrane contour irregularity with preserved nucleus-to-cytoplasm ratio (Fig. 1). HSIL involves the entire epithelium, or the lower two thirds, and is typified by a loss of maturation, nuclear hyperchromasia, and membrane irregularity, as well as a decrease in the nucleus-to-cytoplasm ratio (Fig. 2). Immunoperoxidase staining for p16 can assist in identifying dark nuclear and cytoplasmic staining in HSIL [31].

\section{Diagnosis}

The diagnosis of AIN is made from cytology or biopsy during routine examinations. Anal cytology can be performed at a primary care provider's office, using a swab. If screening is positive for HSIL or LSIL, then patients should be referred for a formal biopsy. Studies examining the sensitivity and specificity of anal cytology vary. The reported sensitivity of anal cytology for the detection of any-grade AIN ranges from 47-90\%, with improving sensitivity for high-grade disease or larger areas of disease $[32,33]$. Specificity for anal cytology is lower, with ranges from $32-60 \%[34,35]$.

In addition to cytology, a visual examination of the anus with a direct rectal exam is an important part of the AIN diagnosis.

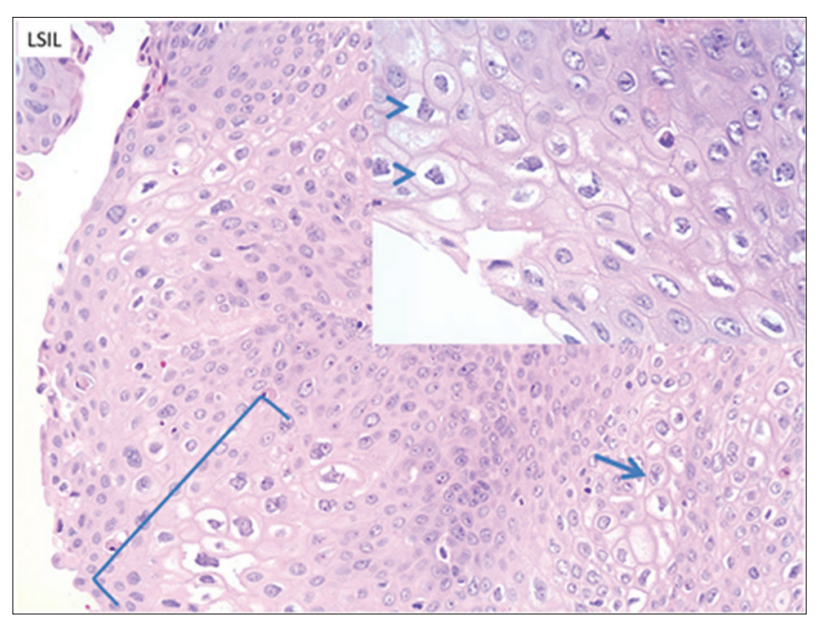

Figure 1 Low-grade squamous intraepithelial lesion (LSIL/AIN-1) Low-grade squamous intraepithelial lesion (LSIL/AIN-1). The lowgrade lesion is typically characterized by marked superficial cell atypia (bracket) with nuclear enlargement and nuclear membrane contour irregularity with preserved nucleus-to-cytoplasm ratio. Bi-nucleation is often seen (arrow). The lower third of the squamous epithelium has more uniform appearance with minmal nuclear variation.

Inset: higher power view of nuclear irregularities, along with perinuclear clearing (koilocytic change, arrowheads)

[Hematoxylin and eosin (H\&E) 400x; inset 600x] 


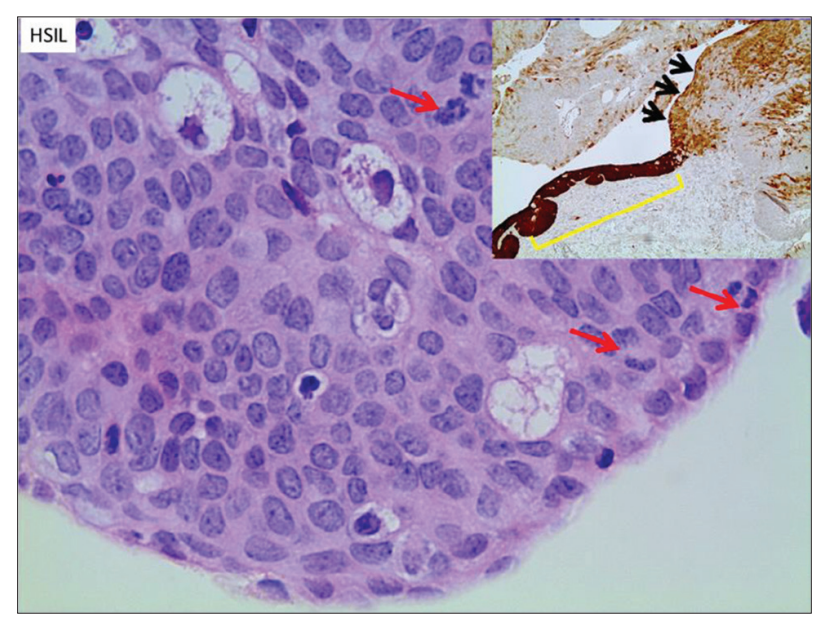

Figure 2 High-grade squamous intraepithelial lesion (HSIL/AIN-2/3) High-grade squamous intraepithelial lesion (HSIL/AIN-2/3). The highgrade lesion exhibits more severe atypia in the lower two-thirds or through the full thickness of the epithelium. There is loss of maturation, and nucleus-to-cytoplasm ratio is decreased. Nuclear membrane contours are irregular and chromatin is hyperchromatic. Disorganized growth is also noted. Increased mitotic activity in the mid to superficial aspects of the epithelium is a feature of HSIL (arrows).

Inset: Immunoperoxidase stain for p16 a proxy test for presence of HPV. Diffuse dark nuclear and cytoplasmic staining is characteristic of HSIL (AIN2/3, bracket). Variable, mainly cytoplasmic staining is more typical of the LSIL as seen adjacent to the HSIL in this case (arrows). [H\&E $600 \mathrm{x}$; inset $50 \mathrm{x}$ ]

Changes in sphincter tone or irregularities of the mucosa can indicate potential lesions that may need to be biopsied. Despite their benefits, visual examination and digital rectal exam alone are not sufficient for the screening or diagnosis of AIN.

Formal biopsy can be performed via conventional anoscopy or high-resolution anoscopy (HRA), and will typically provide sufficient tissue for microscopic evaluation to determine the presence of LSIL or HSIL. An advantage of tissue biopsy is that it provides more architecture and, in some cases, allows for a more definitive diagnosis compared to cytology alone. HRA is a tool that can be utilized in the office to diagnose AIN, as well as for therapeutic intervention [36]. After acetic acid application, a magnifying anoscope is used to examine the anus and lower rectum. The acetic acid will cause dysplastic cells to be more visible compared with surrounding tissue; this is of assistance in a targeted biopsy for pathologic assessment.

\section{Screening}

The appropriate screening protocol for low- or highgrade AIN is not standardized and remains controversial. Proponents of screening stress that detection and treatment of AIN can prevent the premalignant lesion from progressing to anal cancer. The benefits of screening are clear in cervical cancer and its application in anal cancer seems appropriate, given its similarity to cervical cancer in terms of pathophysiology.
Despite this corollary, there are no official screening guidelines for AIN from the United States Preventive Services Task Force (USPSTF), or from any major gastrointestinal medicine or surgical society [37].

\section{Low-risk}

Low-risk individuals are those who are immunocompetent, HIV-negative, non-MSM, without IBD, and with no history of cervical cancer. These individuals are not routinely screened with any modality. No evidence exists that these individuals should be screened, given the low incidence of AIN or anal cancer within these populations. They would benefit from an examination if they developed worrisome signs for AIN or anal cancer, such as palpable masses, rectal discharge, warts, or bleeding.

\section{High-risk}

High-risk patients are those who are HIV-positive, MSM, or immunocompromised. Despite the lack of guidelines from the major gastrointestinal medical or surgical societies, various infectious disease societies have recommended annual cytology for HIV-positive patients, especially those who are MSM, or have a history of cervical cancer [38]. A large retrospective review from 2015 showed that patients followed with standard anoscopy or HRA every 3-12 months had lower rates of progression of AIN to anal cancer, compared to epidemiologic data $[39,40]$. The ASCRS Standard Committee from 2012 recognized that cytology was being used for screening high-risk patients, although concern was raised about the high false negative rates reported in HIV-positive patients. Nevertheless, they agreed with the infectious disease societies and recommended cytology for high-risk patients with referral to a specialist for anoscopy if positive [41]. Screening has been found to be cost-effective for AIN, but its implementation remains variable [42].

The optimal screening tool for high risk patients is debated. Cytology is relatively easy and can be done in a primary care physician's office; however, as stated above, it can have a high false negative rate for high-risk patients. Given this concern, some have advocated that high-risk patients should undergo HRA for screening, given its better sensitivity and specificity as well as the ability to treat at the time of screening $[43,44]$. The major disadvantages of this strategy are 1) there may not be enough caregivers who can do HRA, and 2) the high cost of screening with this modality.

There is no established consensus for the timing of subsequent surveillance, though most societies recommend yearly surveillance for HIV-positive males, and every 3-6 months for those with low- or high-grade AIN. There have been no prospective studies examining the timeframe of follow up and, given the variable nature of AIN development into anal cancer, it is difficult to form established standardized guidelines. 


\section{Treatment}

Surgical excision was preferred in the past before the wide availability of local and targeted therapies. There is little debate that AIN can develop into anal cancer, but the timing and optimal treatment are not standardized. Management options are often separated into surveillance (watchful waiting), or more aggressive interventional strategies. The current literature lacks major randomized studies and most supportive data come from single institutions, case control series or case reports [45]. Treatment options for low- or high-grade lesions include excision, fulguration, laser therapy, or topical treatments such as trichloroacetic acid (TCA), imiquimod or 5-fluorouracil (5-FU). Given the wide array of treatments and evolving literature, specialists with expertise in AIN should be the ones to provide care for these patients.

In the past, surgical excision was the treatment of choice for the majority of clinicians $[38,46]$. Despite this aggressive treatment, patients can still have recurrences, while repeated procedures increase the risk of stenosis or fecal incontinence, especially in patients with circumferential disease. Cases of recurrence or incomplete excision occur more commonly in patients with multifocal lesions or those with a significant disease burden. The rate of recurrence reported after wide local excision in the literature has a large range, between 9-63\% [41]. Given these findings, some have suggested surveillance of high-risk lesions; chemoradiotherapy after the development of anal cancer would then be the optimal treatment [46]. No studies comparing the morbidity or mortality of these 2 strategies have been performed.

Fulguration with electrocautery can be carried out during HRA or anoscopy. Initial studies showed favorable results with no recurrence of disease for HIV-negative patients with high-grade AIN. The recurrence rate for HIV-positive patients, however, was $80 \%$ at 2 -year follow up [47]. Subsequent studies found that a third of HIV-positive patients have a complete response to fulguration, with another third having a partial response, and the remainder no response. Their recurrence rate at 30 months was $25 \%$ and the response was dependent on the quantity of fulgurations [48].

Infrared or laser coagulation may offer treatment of AIN with decreased morbidity. It can be done in the office and is tolerated well by the majority of patients. Infrared and laser coagulation primarily target superficial lesions with limited penetration of deeper tissues, allowing patients to return to daily activities sooner than with fulguration [49]. This treatment is not FDA-approved for AIN, but preliminary studies show some benefit. Retrospective data show that patients serially followed and treated with infrared coagulation, did not have progression to anal cancer; recurrence of AIN remains a concern, however, especially in HIV-positive patients. The recurrence rate for HIVpositive patients was $61 \%$ at 1 year, compared to $38 \%$ in HIVnegative patients [50]. Overall, this treatment could be effective, but more prospective data and standardization are required.

Topical therapies offer an alternative to more invasive procedures. The primary topical agents are TCA, imiquimod, and 5-FU. TCA has a good safety profile with few major side effects. It can be applied during examination, and is well- tolerated. Retrospective studies show that TCA can induce clearance of high-grade AIN in $71-79 \%$ of patients, but may not be effective for extensive or bulky disease [51]. 5-FU is a chemotherapy agent that inhibits DNA synthesis and, when applied topically, can clear AIN. Rates of clearance vary, but prospective data showed a complete clearance of $90 \%$, with a recurrence rate of $50 \%$ at 6 months [52]. Side effects include hypopigmentation or skin irritation. Imiquimod (trade name Aldara) is a synthetic immune modulator that upregulates a patient's innate immune system to include antiviral activity. For HIV-positive patients, imiquimod has been shown to downgrade high-risk lesions to low-risk lesions in randomized trials [53], and $61 \%$ of patients in a randomized study had absence of high-grade lesions with imiquimod treatment [54].

The combination of topical and invasive procedures could offer the best benefit for treatment of AIN. At one large single center, 248 patients were followed with exams and serial ablative procedures under HRA and supplemental topical treatments. The authors were able to demonstrate $80 \%$ clearance of highgrade dysplasia, and only $1.2 \%$ of the patients developed anal cancer [55].

\section{HPV vaccination}

HPV vaccination may prove to be a prevention tool for AIN. The quadrivalent vaccine is effective for high-risk HPV strains $6,11,16$, and 18 and has been previously proven safe and effective in the prevention of cervical cancer. Emerging studies indicate that vaccination may be the most realistic long-term approach to prevention and possible treatment of AIN. The benefit of the vaccine will depend on the timing of vaccination and exposure to HPV [23].

Administration of the vaccine after the diagnosis of AIN in order to assist with prevention in the future has been studied. Retrospective data from a large Swedish trial showed that, in MSM with recurrent AIN, those who received the vaccination had significantly lower rates of high-grade AIN moving forward. To further study the therapeutic benefit, a study from 2011 randomized MSM to receive the HPV vaccine vs. placebo. They found the rates of AIN were lower in the vaccine group (13 vs. 17.5 per 100 person years), while they also had a significantly lower risk for persistent HPV infection following vaccination. The benefits of these results seen in MSM can also be applied to heterosexual men and women, given the biological similarity between anal cancer in men and women and the same pathogenesis due to HPV infection [56]. The administration of the vaccine to young people prior to sexual activity appears to provide the greatest benefit for prevention of AIN and anal cancer [57].

\section{Concluding remarks}

AIN is a premalignant lesion for anal cancer. It is more commonly found in high-risk patients (e.g., HPV/HIV 
infections, post-organ transplantation patients and MSM) and the development of AIN is driven by HPV infection. There still remains significant controversy regarding screening, surveillance and treatment for AIN, but emerging data suggest that close patient follow up with a combination of ablative and topical treatments may offer the greatest benefit.

\section{Acknowledgment}

The authors would like to thank Mary Kwatkosky-Lawlor for her assistance in the editing and submission of this article.

\section{References}

1. Darragh TM, Colgan TJ, Thomas Cox J, et al; Members of the LAST Project Work Groups. The Lower Anogenital Squamous Terminology Standardization project for HPV-associated lesions: background and consensus recommendations from the College of American Pathologists and the American Society for Colposcopy and Cervical Pathology. Int J Gynecol Pathol 2013;32:76-115.

2. Roberts JR, Siekas LL, Kaz AM. Anal intraepithelial neoplasia: a review of diagnosis and management. World J Gastrointest Oncol 2017;9:50-61.

3. Stanley MA, Winder DM, Sterling JC, Goon PK. HPV infection, anal intra-epithelial neoplasia (AIN) and anal cancer: current issues. BMC Cancer 2012;12:398.

4. Johnson LG, Madeleine MM, Newcomer LM, Schwartz SM, Daling JR. Anal cancer incidence and survival: the surveillance, epidemiology, and end results experience, 1973-2000. Cancer 2004;101:281-288.

5. Nielsen A, Munk C, Kjaer SK. Trends in incidence of anal cancer and high-grade anal intraepithelial neoplasia in Denmark, 19782008. Int J Cancer 2012;130:1168-1173.

6. Scholefield JH, Castle MT, Watson NF. Malignant transformation of high-grade anal intraepithelial neoplasia. Br J Surg 2005;92:1133-1136.

7. Watson AJ, Smith BB, Whitehead MR, Sykes PH, Frizelle FA. Malignant progression of anal intra-epithelial neoplasia. ANZ J Surg 2006;76:715-717.

8. Machalek DA, Poynten M, Jin F, et al. Anal human papillomavirus infection and associated neoplastic lesions in men who have sex with men: a systematic review and meta-analysis. Lancet Oncol 2012;13:487-500.

9. Tong WW, Jin F, McHugh LC, et al. Progression to and spontaneous regression of high-grade anal squamous intraepithelial lesions in HIV-infected and uninfected men. AIDS 2013;27:2233-2243.

10. Centers for Disease Control and Prevention. Sexually Transmitted Disease Surveillance 2015. Other Sexually Transmitted Diseases 2015. Atlanta: US Department of Health and Human Services. https://www.cdc.gov/std/stats [Accessed 4 February 2019].

11. Dempsey AF. Human papillomavirus: the usefulness of risk factors in determining who should get vaccinated. Rev Obstet Gynecol 2008; 1:122-128

12. Crosbie EJ, Einstein MH, Franceschi S, Kitchener HC. Human papillomavirus and cervical cancer. Lancet 2013;382:889-899.

13. Bosch FX, Broker TR, Forman D, et al; authors of ICO Monograph Comprehensive Control of HPV Infections and Related Diseases Vaccine Volume 30, Supplement 5, 2012. Comprehensive control of human papillomavirus infections and related diseases. Vaccine 2013;31 Suppl 7:H1-H31.
14. De Vuyst H, Clifford GM, Nascimento MC, Madeleine MM, Franceschi S. Prevalence and type distribution of human papillomavirus in carcinoma and intraepithelial neoplasia of the vulva, vagina and anus: a meta-analysis. Int J Cancer 2009;124:1626-1636.

15. Salit IE, Tinmouth J, Chong S, et al. Screening for HIV-associated anal cancer: correlation of HPV genotypes, p16, and E6 transcripts with anal pathology. Cancer Epidemiol Biomarkers Prev 2009;18:1986-1992.

16. Gami B, Kubba F, Ziprin P. Human papilloma virus and squamous cell carcinoma of the anus. Clin Med Insights Oncol 2014;8:113-119.

17. Kovi J, Tillman RL, Lee SM. Malignant transformation of condyloma acuminatum. A light microscopic and ultrastructural study. Am J Clin Pathol 1974;61:702-710.

18. Zaki SR, Judd R, Coffield LM, Greer P, Rolston F, Evatt BL. Human papillomavirus infection and anal carcinoma. Retrospective analysis by in situ hybridization and the polymerase chain reaction. Am J Pathol 1992;140:1345-1355.

19. Münger K, Baldwin A, Edwards KM, et al. Mechanisms of human papillomavirus-induced oncogenesis. J Virol 2004;78:11451-11460.

20. Medford RJ, Salit IE. Anal cancer and intraepithelial neoplasia: epidemiology, screening and prevention of a sexually transmitted disease. CMAJ 2015;187:111-115.

21. Frisch M, Fenger C, van den Brule AJ, et al. Variants of squamous cell carcinoma of the anal canal and perianal skin and their relation to human papillomaviruses. Cancer Res 1999;59:753-757.

22. Wasserman P, Rubin DS, Turett G. Review: Anal intraepithelial neoplasia in HIV-infected men who have sex with men: is screening and treatment justified? AIDS Patient Care STDS 2017;31:245-253.

23. Park IU, Palefsky JM. Evaluation and management of anal intraepithelial neoplasia in HIV-negative and HIV-positive men who have sex with men. Curr Infect Dis Rep 2010;12:126-133.

24. Maranga IO, Hampson L, Oliver AW, et al. HIV infection alters the spectrum of HPV subtypes found in cervical smears and carcinomas from Kenyan women. Open Virol J 2013;7:19-27.

25. Bedimo RJ, McGinnis KA, Dunlap M, Rodriguez-Barradas MC, Justice AC. Incidence of non-AIDS-defining malignancies in HIVinfected versus noninfected patients in the HAART era: impact of immunosuppression. J Acquir Immune Defic Syndr 2009;52:203-208.

26. Engels EA, Biggar RJ, Hall HI, et al. Cancer risk in people infected with human immunodeficiency virus in the United States. Int $J$ Cancer 2008;123:187-194.

27. Patel HS, Silver AR, Northover JM. Anal cancer in renal transplant patients. Int J Colorectal Dis 2007;22:1-5.

28. Madeleine MM, Finch JL, Lynch CF, Goodman MT, Engels EA. HPV-related cancers after solid organ transplantation in the United States. Am J Transplant 2013;13:3202-3209.

29. Wisniewski A, Fléjou JF, Siproudhis L, Abramowitz L, Svrcek M, Beaugerie L. Anal neoplasia in inflammatory bowel disease: classification proposal, epidemiology, carcinogenesis, and risk management perspectives. J Crohns Colitis 2017;11:1011-1018.

30. Slesser AA, Bhangu A, Bower M, Goldin R, Tekkis PP. A systematic review of anal squamous cell carcinoma in inflammatory bowel disease. Surg Oncol 2013;22:230-237.

31. Abbasakoor F, Boulos PB. Anal intraepithelial neoplasia. Br J Surg 2005;92:277-290.

32. Palefsky JM, Holly EA, Hogeboom CJ, Berry JM, Jay N, Darragh TM. Anal cytology as a screening tool for anal squamous intraepithelial lesions. J Acquir Immune Defic Syndr Hum Retrovirol 1997; 14:415-422.

33. Nathan M, Singh N, Garrett N, Hickey N, Prevost T, Sheaff M. Performance of anal cytology in a clinical setting when measured against histology and high-resolution anoscopy findings. AIDS 2010;24:373-379.

34. Leeds IL, Fang SH. Anal cancer and intraepithelial neoplasia screening: A review. World J Gastrointest Surg 2016;8:41-51. 
35. Fox PA, Seet JE, Stebbing J, et al. The value of anal cytology and human papillomavirus typing in the detection of anal intraepithelial neoplasia: a review of cases from an anoscopy clinic. Sex Transm Infect 2005;81:142-146.

36. Palefsky JM. Practising high-resolution anoscopy. Sex Health 2012;9:580-586.

37. Alam NN, Narang SK, Köckerling F, Daniels IR, Smart NJ. Anal Sphincter Augmentation Using Biological Material. Front Surg 2015;2:60.

38. Dindo D, Nocito A, Schettle M, Clavien PA, Hahnloser D. What should we do about anal condyloma and anal intraepithelial neoplasia? Results of a survey. Colorectal Dis 2011;13:796-801.

39. Long KC, Menon R, Bastawrous A, Billingham R. Screening, surveillance, and treatment of anal intraepithelial neoplasia. Clin Colon Rectal Surg 2016;29:57-64.

40. Crawshaw BP, Russ AJ, Stein SL, et al. High-resolution anoscopy or expectant management for anal intraepithelial neoplasia for the prevention of anal cancer: is there really a difference? Dis Colon Rectum 2015;58:53-59.

41. Steele SR, Varma MG, Melton GB, Ross HM, Rafferty JF, Buie WD; Standards Practice Task Force of the American Society of Colon and Rectal Surgeons. Practice parameters for anal squamous neoplasms. Dis Colon Rectum 2012;55:735-749.

42. Goldie SJ, Kuntz KM, Weinstein MC, Freedberg KA, Palefsky JM. Cost-effectiveness of screening for anal squamous intraepithelial lesions and anal cancer in human immunodeficiency virus-negative homosexual and bisexual men. Am J Med 2000;108:634-641.

43. Dalla Pria A, Alfa-Wali M, Fox P, et al. High-resolution anoscopy screening of HIV-positive MSM: longitudinal results from a pilot study. AIDS 2014;28:861-867.

44. Nahas CS, de Silvia Filho EV, Segurado AA, et al. Screening anal dysplasia in HIV-infected patients: is there an agreement between anal pap smear and high-resolution anoscopy-guided biopsy? Dis Colon Rectum 2009;52:1854-1860.

45. Weis SE. Current treatment options for management of anal intraepithelial neoplasia. Onco Targets Ther 2013;6:651-665.

46. Cleary RK, Schaldenbrand JD, Fowler JJ, Schuler JM, Lampman RM. Treatment options for perianal Bowen's disease: survey of American Society of Colon and Rectal Surgeons Members. Am Surg 2000;66:686-688.

47. Chang GJ, Berry JM, Jay N, Palefsky JM, Welton ML. Surgical treatment of high-grade anal squamous intraepithelial lesions: a prospective study. Dis Colon Rectum 2002;45:453-458.

48. Burgos J, Curran A, Landolfi S, et al. The effectiveness of electrocautery ablation for the treatment of high-grade anal intraepithelial neoplasia in HIV-infected men who have sex with men. HIV Med 2016;17:524-531.

49. Goldstone SE, Kawalek AZ, Huyett JW. Infrared coagulator: a useful tool for treating anal squamous intraepithelial lesions. Dis Colon Rectum 2005;48:1042-1054.

50. Goldstone RN, Goldstone AB, Russ J, Goldstone SE. Long-term follow-up of infrared coagulator ablation of anal high-grade dysplasia in men who have sex with men. Dis Colon Rectum 2011;54:1284-1292.

51. Megill C, Wilkin T. Topical therapies for the treatment of anal high-grade squamous intraepithelial lesions. Semin Colon Rectal Surg 2017;28:86-90.

52. RichelO, WielandU,deVriesHJ, etal.Topical5-fluorouraciltreatment of anal intraepithelial neoplasia in human immunodeficiency viruspositive men. Br J Dermatol 2010;163:1301-1307.

53. Wieland U, Brockmeyer NH, Weissenborn SJ, et al. Imiquimod treatment of anal intraepithelial neoplasia in HIV-positive men. Arch Dermatol 2006;142:1438-1444.

54. Fox PA, Nathan M, Francis N, et al. A double-blind, randomized controlled trial of the use of imiquimod cream for the treatment of anal canal high-grade anal intraepithelial neoplasia in HIV-positive MSM on HAART, with long-term follow-up data including the use of open-label imiquimod. AIDS 2010;24:2331-2335.

55. Pineda CE, Berry JM, Jay N, Palefsky JM, Welton ML. Highresolution anoscopy targeted surgical destruction of anal highgrade squamous intraepithelial lesions: a ten-year experience. Dis Colon Rectum 2008;51:829-835.

56. Palefsky JM, Giuliano AR, Goldstone S, et al. HPV vaccine against anal HPV infection and anal intraepithelial neoplasia. N Engl J Med 2011;365:1576-1585.

57. Stier EA, Chigurupati NL, Fung L. Prophylactic HPV vaccination and anal cancer. Hum Vaccin Immunother 2016;12:1348-1351. 